\title{
A First Study of Environmental Noise Coupling to the Virgo Interferometer
}

\author{
F Acernese ${ }^{6}, \mathbf{P}$ Amico $^{10}$, M Al-Shourbagy ${ }^{11}$, S Aoudia $^{7}$, \\ S Avino ${ }^{6}$, D Babusci ${ }^{4}$, G Ballardin ${ }^{2}, \mathbf{R}$ Barillé $^{2}$, F Barone $^{6}$, \\ L Barsotti $^{11}$, M Barsuglia ${ }^{8}$, F Beauville $^{1}$, M A Bizouard ${ }^{8}$, \\ C Boccara ${ }^{9}$, F Bondu ${ }^{7}$, L Bosi $^{10}$, C Bradaschia ${ }^{11}$, \\ S Braccini $^{11}$, A Brillet ${ }^{7}, \mathbf{V}$ Brisson $^{8}$, L Brocco ${ }^{12}$, \\ D Buskulic ${ }^{1}$, E Calloni ${ }^{6}$, E Campagna ${ }^{3}$, F Cavalier ${ }^{8}$, \\ R Cavalieri ${ }^{2}$, G Cella ${ }^{11}$, E Chassande-Mottin ${ }^{7}, \mathbf{C}$ Corda $^{11}$, \\ A C Clapson ${ }^{8}, \mathbf{F}$ Cleva $^{7}, \mathbf{J}$ P Coulon ${ }^{7}, \mathbf{E} \mathbf{C u o c o}^{2}$, \\ V Dattilo ${ }^{2}$, M Davier ${ }^{8}, \mathbf{R}$ De Rosa ${ }^{6}, \mathbf{L}$ Di Fiore ${ }^{6}$, \\ A Di Virgilio ${ }^{11}$, B Dujardin ${ }^{7}$, A Eleuteri ${ }^{6}$, D Enard ${ }^{2}$, \\ I Ferrante $^{11}$, F Fidecaro ${ }^{11}$, I Fiori ${ }^{11}$, R Flaminio Fl, $^{1,2}$, \\ J D Fournier ${ }^{7}, \mathbf{S}$ Frasca $^{12}$, F Frasconi ${ }^{2,11}$, A Freise $^{2}$, \\ L Gammaitoni ${ }^{10}$, A Gennai ${ }^{11}$, A Giazotto ${ }^{11}$, G Giordano ${ }^{4}$, \\ L Giordano ${ }^{6}$, R Gouaty ${ }^{1}$, D Grosjean ${ }^{1}$, G Guidi ${ }^{3}$, S Hebri $^{2}$, \\ H Heitmann ${ }^{7}$, P Hello ${ }^{8}$, L Holloway ${ }^{2}$, S Kreckelbergh ${ }^{8}$, \\ P La Penna ${ }^{2}$, V Loriette ${ }^{9}$, M Loupias ${ }^{2}, \mathbf{G}$ Losurdo $^{3}$, \\ J M Mackowski ${ }^{5}$, E Majorana ${ }^{12}, \mathbf{C} \mathbf{N}$ Man $^{7}$, \\ M Mantovani ${ }^{11}$, F Marchesoni ${ }^{10}, \mathbf{F}$ Marion $^{1}, \mathbf{J ~ M a r q u e}^{2}$, \\ F Martelli ${ }^{3}$, A Masserot ${ }^{1}$, M Mazzoni ${ }^{3}$, L Milano ${ }^{6}$, \\ C Moins ${ }^{2}$, J Moreau ${ }^{9}, \mathbf{N}$ Morgado $^{5}$, B Mours ${ }^{1}$, A Pai ${ }^{12}$, \\ C Palomba ${ }^{12}$, F Paoletti ${ }^{2,11}$, S Pardi ${ }^{6}$, A Pasqualetti ${ }^{2}$, \\ R Passaquieti ${ }^{11}$, D Passuello ${ }^{11}$, B Perniola ${ }^{3}$, \\ F Piergiovanni ${ }^{3}$ L Pinard $^{5}$, R Poggiani ${ }^{11}$, M Punturo ${ }^{10}$, \\ P Puppo ${ }^{12}$, K Qipiani ${ }^{6}$, P Rapagnani $^{12}$, V Reita ${ }^{9}$, \\ A Remillieux ${ }^{5}$, F Ricci ${ }^{12}$, I Ricciardi ${ }^{6}$, P Ruggi ${ }^{2}$, G Russo ${ }^{6}$, \\ S Solimeno $^{6}$, A Spallicci ${ }^{7}, \mathbf{R}$ Stanga $^{3}, \mathbf{R}$ Taddei $^{2}$, \\ D Tombolato ${ }^{1}$, M Tonelli ${ }^{11}$, A Toncelli ${ }^{11}$, E Tournefier ${ }^{1}$, \\ F Travasso $^{10}, \mathbf{G}$ Vajente $^{11}, \mathbf{D}$ Verkindt $^{1}, \mathbf{F}$ Vetrano $^{3}$, \\ A Viceré ${ }^{3}$, J Y Vinet ${ }^{7}, \mathbf{H}$ Vocca $^{10}, \mathbf{M}$ Yvert $^{1}$ and $Z$ Zhang $^{2}$ \\ ${ }^{1}$ Laboratoire d'Annecy-le-Vieux de Physique des Particules, 74941 \\ Annecy-le-Vieux, France; \\ 2 European Gravitational Observatory (EGO), 56021 Cascina (PI) Italy; \\ ${ }^{3}$ INFN Sez. di Firenze/Urbino and/or Università di Firenze, 50019 Sesto \\ Fiorentino, and/or Università di Urbino, 61019 Urbino, Italy; \\ ${ }^{4}$ INFN, Laboratori Nazionali di Frascati, 00044 Frascati (RM) Italy; \\ ${ }^{5}$ LMA, 69622 Villeurbanne, Lyon, France; \\ ${ }^{6}$ INFN Sez. di Napoli and/or Università di Napoli "Federico II", 80126 Napoli, \\ and/or Università di Salerno, 84084 Fisciano (SA), Italy; \\ 7 Observatoire de la Côte d'Azur, 06034 Nice, France;
}

Corresponding author: I. Fiori, INFN Sez. di Pisa, Edificio C, Polo Fibonacci, Largo B Pontecorvo 3, 56127 Pisa, Italy (Irene.Fiori@pi.infn.it). 
8 Laboratoire de l'Accélérateur Linéaire and/or CNRS-IN2P3 and Université de Paris Sud, 91898 Orsay, France;

${ }^{9}$ ESPCI, 75005 Paris, France;

10 INFN Sez. di Perugia and/or Università di Perugia, 06123 Perugia, Italy;

11 INFN Sez. di Pisa and/or Università di Pisa, 56127 Pisa, Italy;

12 INFN Sez. di Roma and/or Università di Roma "La Sapienza", 00185 Roma, Italy.

\begin{abstract}
During the commissioning of the Virgo interferometer a search for environmental noise contributions to the dark fringe signal was undertaken. Dedicated tests have been performed to identify major sources of disturbances and to understand the coupling mechanism with the interferometer. The major effect is due to seismic/acoustic noise coupling to the laser beam before the input mode cleaner, then propagating as beam power noise to the ITF dark fringe output signal. In this paper we illustrate the tests performed and preliminary results of our investigation.

PACS number: 04.80.Nn
\end{abstract}

Some figures in this article appear in color only in the electronic version.

\title{
1. Introduction
}

During the first year of commissioning activity the Virgo interferometer has been run in various configurations of increasing complexity: independent $\mathrm{FP}$ cavities (runs $\mathrm{C} 1$ to $\mathrm{C} 3$ ), recombined mode (runs $\mathrm{C} 3$ and $\mathrm{C} 4$ ) and finally recombined with power recycling mode (run C5). Throughout the runs the detector sensitivity has steadily improved as the several control systems (i.e. ITF locking, beam alignment, laser frequency stabilization, mirrors and mirror suspensions damping) first implemented for the short CITF [1] have been adapted to Virgo and constantly improved. For each run the major noise source limiting the sensitivity have been analyzed and understood $[2,3]$.

As part of the noise hunting, we carried out an investigation for the presence of effects from noise of environmental origin (namely seismic, acoustic and electromagnetic) in the Virgo sensitivity.

During C1-C3 runs a coherency analysis between the dark fringe signal and external environmental probes (seismometers, microphones and magnetometers) revealed the presence of seismic/acoustic disturbances in the ITF readout signal, and indicated our laser light injection system as their most likely entry point. In addition, dedicated tests have been carried out which allowed to identify as noise sources mechanical devices such as pumps, air conditioning machines, electronics racks.

During $\mathrm{C} 4$ we tested the robustness of our ITF by generating an intense broadband acoustic noise in the clean room housing our laser and injection system. The analysis of these data allowed to understand better the coupling mechanism and to track a path of the noise down to the dark fringe.

In Sec. 2 of this paper we describe the results of coherency analysis and illustrate one test performed for identifying noise sources. In Sec. 3 we describe the acoustic noise injection test and the measured effects. Data are analyzed in Sec. 4 to track a path for the injected noise down to the dark fringe signal. Finally we compute the effect of the produced power noise on the Virgo sensitivity curve. 


\section{Coherency analysis and sources identification}

The Virgo environment is monitored by several seismic, acoustic and electromagnetic probes located at most crucial points along the ITF $[4,5]$. A practical method to reveal if seismic, acoustic or electromagnetic noises are affecting or limiting the detector sensitivity, is to compute the multi-coherence function [6] between the environmental channels and the ITF readout channel (the dark fringe). The multi-coherence tool is implemented in the Virgo noise analysis software library (NAP) and explained in detail in reference [7]. Essentially, the cross correlation between the dark fringe and each environmental signal is subtracted, properly accounting for the correlations among environmental channels. Multi-coherence is the proper tool to use in the case of environmental noises, which are naturally coupled (as for example are seismic and acoustic noises) and therefore sensors signals can be significantly correlated. In the hypothesis of linear coupling to the dark fringe, the coherency function also provides a measurement of the size of the noise contribution as function of frequency. In addition, the physical location of the probe which senses the highest coherence helps to locate the noisy sources.

We performed this analysis on data of four commissioning runs ( $\mathrm{C} 1$ to $\mathrm{C} 4$ ). Results are shown in Figure 1. For simplicity, multi-coherences were evaluated using only the one seismometer, microphone and magnetometer which among all had the highest coherence with the dark fringe signal. These turned out to be always the probes located within and nearby the clean room housing our laser and light injection system, indicating it as the likely location where the noise couples to the ITF.

At this level of sensitivity the electromagnetic noise contribution is limited to harmonics of the mains power line $(50 \mathrm{~Hz}$ in Italy). Herein the coupling path of this noise has not been investigated further. In the following we concentrate on the seismic/acoustic noise that appears to be limiting the sensitivity at various frequency locations within the ITF detection bandwidth.

This noise mainly originates from vibrations of mechanical devices such as vacuum pumps, air conditioning machines and electronic racks. Several sources have been identified by switching them temporarily off. Figure 2 illustrates the effects recorded during one test performed during run C3 (ITF in single arm configuration). The test consisted in sweeping from $600 \mathrm{~Hz}$ to $400 \mathrm{~Hz}$ (in a time scale of about 5 minute) the rotation frequency of the turbo-molecular pump which preserves the UHV level inside the vacuum tower vessel housing the Mode Cleaner (MC) input bench (IB) suspension [8]. The test allows to clearly identify a family of frequency lines $(600 \mathrm{~Hz}$ and higher harmonics) which are coherently sweeping in the dark fringe and in the seismic sensor nearby the IB suspension tower. Where possible, actions have been taken to reduce the noise produced: i.e. improving the seismic/acoustic isolation of the vacuum pumps, or switching the air conditioning to a lower power regime.

During runs $\mathrm{C} 1, \mathrm{C} 2$ and $\mathrm{C} 3$ the acoustic/seismic noise was propagating to the dark fringe mainly as laser frequency noise (LFN) $[2,3]$. In C2 the disappearing of the large acoustic noise coupling at approximately $10 \mathrm{~Hz}$ (Figure 1, top) corresponds to the reduction of LFN achieved with an improved design of the MC alignment control feedbacks. The acoustic/seismic noise significantly reduces in C3 when the ITF is operated in the recombined mode (Figure 1, bottom). In this ITF configuration phase noise common to both FP cavities, such is laser frequency noise, is suppressed by a factor $\simeq 3 \times 10^{-3}$. Finally, in $\mathrm{C} 4$, the laser frequency noise was further reduced by the implementation of the second stage of frequency stabilization [3]. However, some 

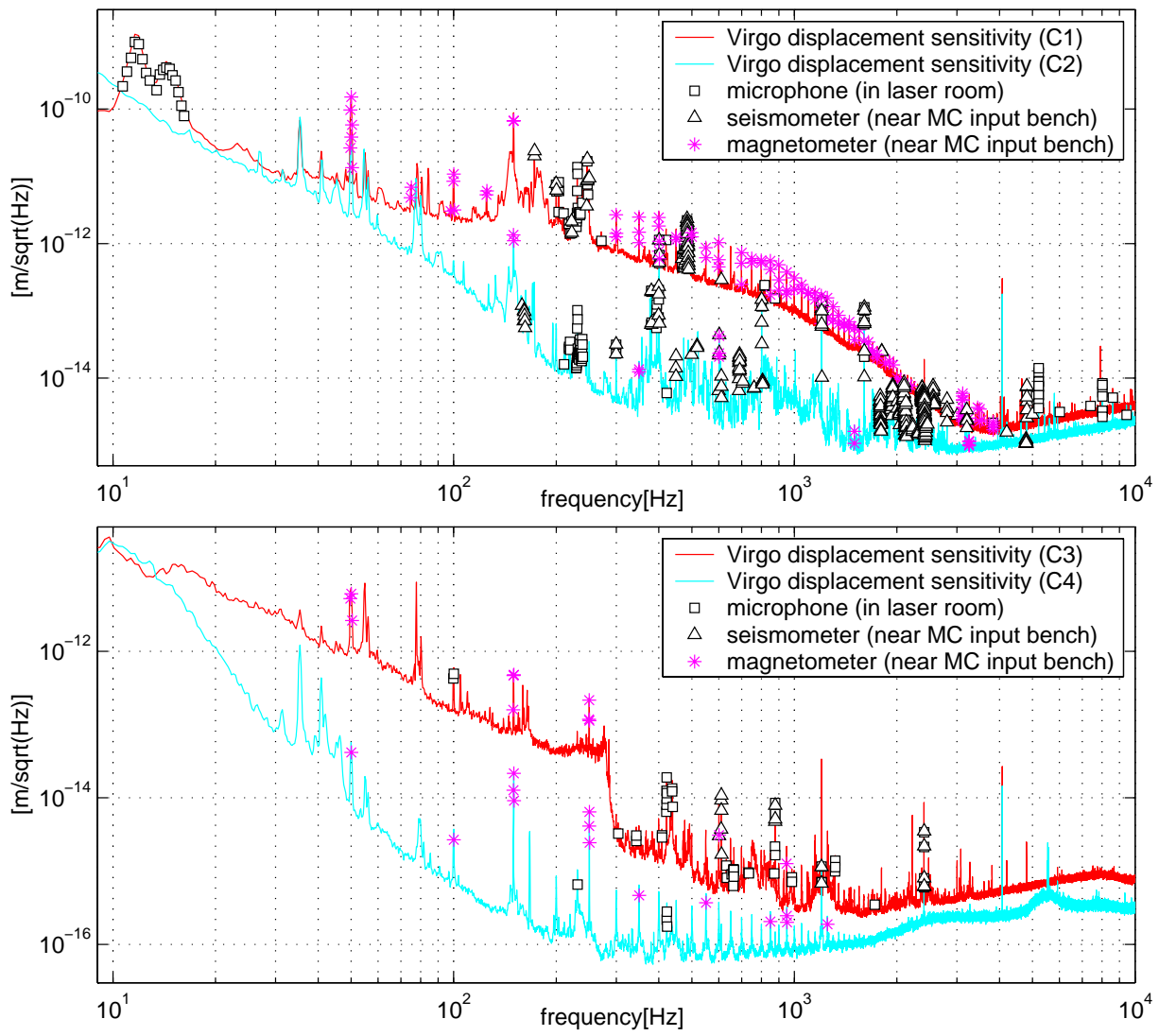

Figure 1. Environmental noise contributions to Virgo sensitivity: (top) runs C1 and $\mathrm{C} 2$, Virgo in single arm configuration; (bottom) runs C3 and C4, Virgo in recombined configuration. Markers indicate the frequencies at which the multicoherence function between the dark fringe photodiode signal and the signals of acoustic (squares), seismic (triangles) or electromagnetic field (stars) sensors is 0.5 or greater. The seismometer response bandwidth is $10 \mathrm{~Hz}$ to $3 \mathrm{kHz}$, the microphone response bandwidth is $1 \mathrm{~Hz}$ to $10 \mathrm{kHz}$.

seismic/acoustic noise is still affecting the $\mathrm{C} 4$ sensitivity at few frequency lines. We investigate further what happened in $\mathrm{C} 4$ in the following two sections.

\section{Test of ITF robustness against acoustic noise}

The analysis described in Sec. 2 indicates the clean room housing the laser, the optics for beam matching, sensors and actuators for input beam alignment as the likely entry point of the acoustic/seismic noise. The room is in air, laser and optics sit on nonsuspended benches. Before entering the ITF, the beam spatial defects as well as its frequency, position and power fluctuations are pre-filtered (cavity pole $f_{c} \simeq 500 \mathrm{~Hz}$ ) by a suspended plane-concave triangular Fabry-Perot cavity (input Mode Cleaner MC) kept under vacuum and working in transmission [10,9]. The MC input bench (IB) carrying the two plane mirrors and the MC curved mirror are both suspended 

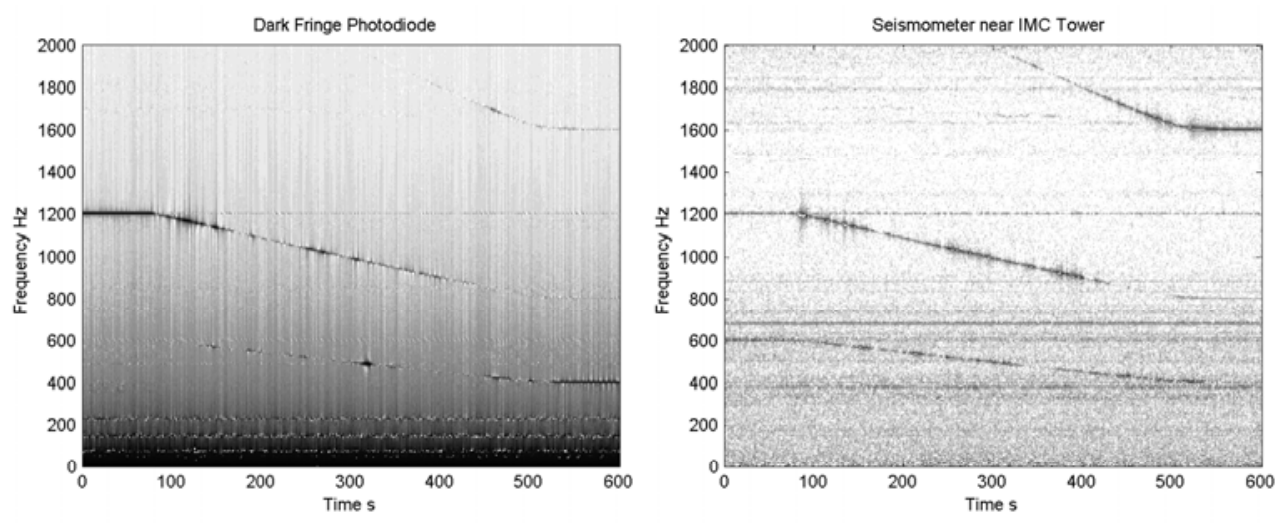

Figure 2. Effect of sweeping down (from $600 \mathrm{~Hz}$ to $400 \mathrm{~Hz}$ ) the rotation frequency of the vacuum pump of the MC input bench suspension tower during commissioning run C3. The pump $1^{\text {st }}, 2^{\text {nd }}$ and $4^{\text {th }}$ harmonics are seen to sweep in the same way in the dark fringe photodiode signal (left) and in the signal from the accelerometer near the tower (right).

from multi-stage pendula (short superattenuators - SA [11]) which exert a passive attenuation of the seismic motion in all degrees of freedom (as $(f / 1 . H z)^{-2 N}$, with $N=4$ for the horizontal d.o.f., $N=2$ for the vertical d.o.f.) above $\approx 1 \mathrm{~Hz}$, while below $1 \mathrm{~Hz}$ the SA internal resonances are actively damped [12]. Several active controls are used to reduce the laser frequency, beam power and beam jitter fluctuations to meet Virgo requirements within the full detection bandwidth. Two feedback controls are devoted to preserve at low frequency $(\leq 10 \mathrm{~Hz})$ the alignment of the laser beam onto the suspended IB, and the alignment of the MC optical axis onto the Virgo North arm axis. The laser power is pre-stabilized at the laser output, and a second stage of stabilization (not active during $\mathrm{C} 4$ ) allows to reduce the power fluctuations of the MC transmitted beam at the level $\frac{\tilde{\delta P}}{P}<10^{-7} / \sqrt{\mathrm{Hz}}$ required to meet Virgo specifications [9].

In order to make a systematic study of the effects produced by acoustic noise, during $\mathrm{C} 4$ we generated noise in the laser room in a controlled and reproducible way, by sending a broadband $(50-4000 \mathrm{~Hz})$ white signal to a loudspeaker. We injected noise with five levels of increasing intensity, up to about 50 times the room acoustic pressure in quiet conditions (Figure 3, top). We observed a corresponding increase of the dark fringe noise up to 10 times the quiet level, in a slightly reduced bandwidth, roughly between $150 \mathrm{~Hz}$ and $1500 \mathrm{~Hz}$ (Figure 3, bottom).

Correspondingly, in the same $150-1500 \mathrm{~Hz}$ bandwidth, we measured an increase of about 10 times of the translational and rotational position fluctuations of the input beam with respect to the MC optical axis, and a similar increase of the fluctuations of the power of the MC transmitted beam to the interferometer, as shown in Figure 4.

In the following section we analyze these data and describe a possible explanation of the observed effects of the acoustic noise injection and of the coupling of the noise to the ITF readout. 

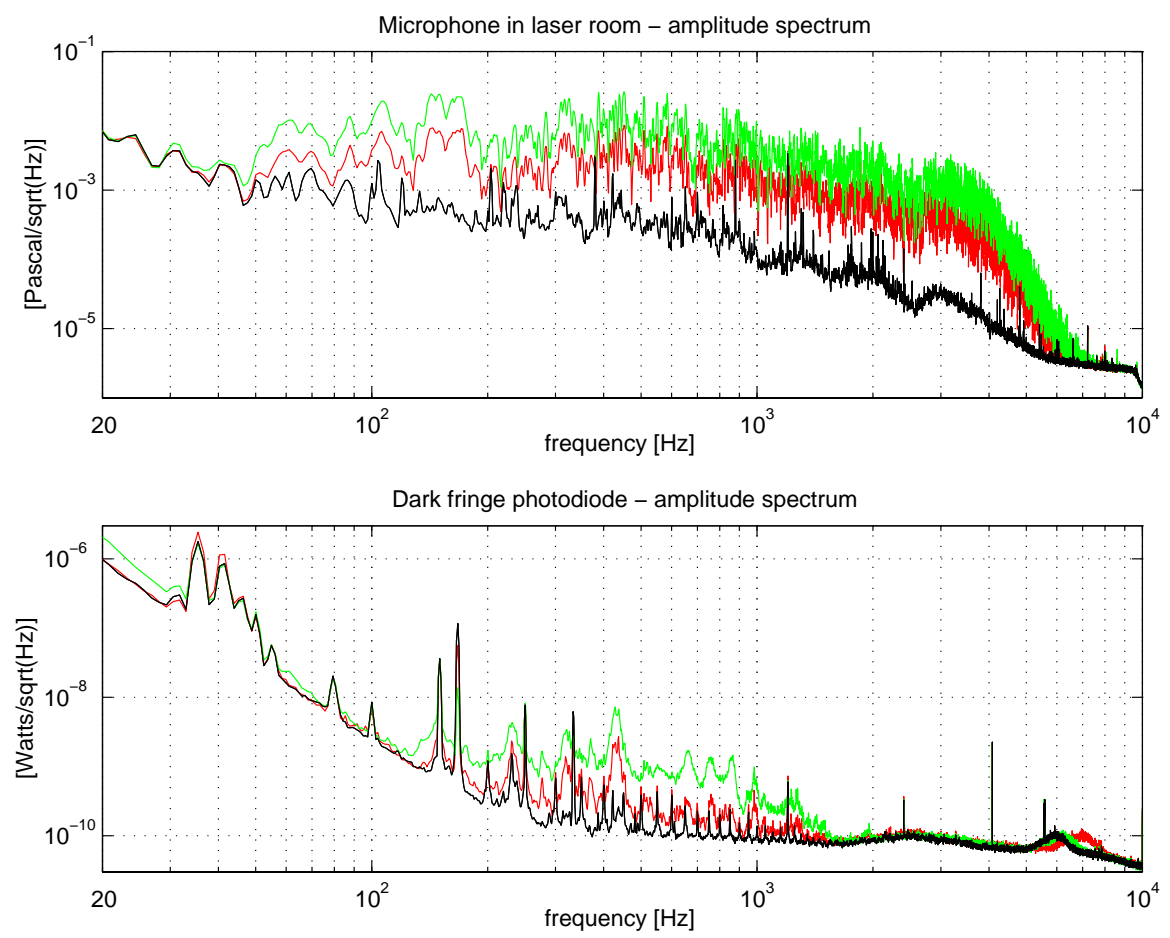

Figure 3. Amplitude spectral densities of the acoustic pressure in the laser room (top) and the dark fringe photodiode signal (bottom): (black) during C4 quiet time operations, i.e. standard acoustic noise in the laser room; colored (or gray) spectra are recorded during the test, corresponding to the highest (green or light gray) and one intermediate (red or dark gray) intensity of the broadband (50-4000 $\mathrm{Hz}$ ) white signal sent to the loudspeaker in the laser room.

\section{A path for the noise to the dark fringe}

\subsection{Some considerations on signals coherency}

The coherency pattern measured between the affected signals allows to track a likely path of the injected acoustic noise to the IFT dark fringe.

The coherency between the microphone and the dark fringe signal is poor and almost absent between $\approx 500 \mathrm{~Hz}$ and $800 \mathrm{~Hz}$ (Figure $5 \mathrm{a}$ ), while good coherence is present over the whole bandwidth between the dark fringe and the photodiode signal measuring the power of the laser beam at ITF input (Figure $5 \mathrm{~b}$ ). This indicates that the coupling occurs through beam power fluctuations which have a quadratic behavior with respect to beam positioning parameters.

A strong correlation exists between the microphone signal and the angular and translational jitters of the input beam with respect to the MC optical axis (Figure 5 $c$ and $d$ ) measured by the error signals of the MC alignment control loop.

We interpret this result as the combined effects of air pressure fluctuations in the laser room and acoustic pressure acting on the beam matching optics causing an increase of the jitter of the laser beam entering the MC.

As we discuss in more detail in the following, these jitters convert into power 

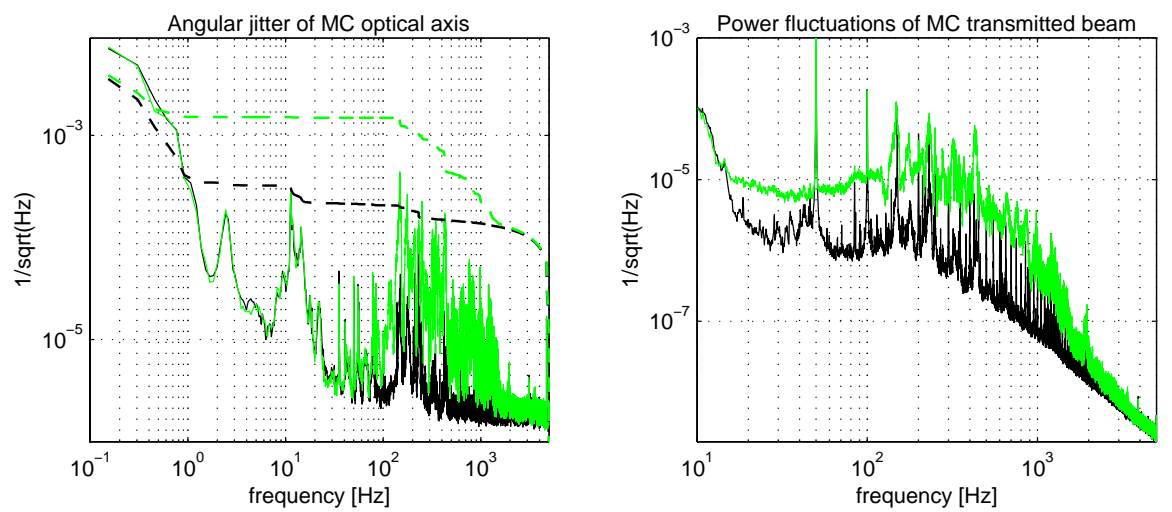

Figure 4. Amplitude spectral densities measured during $\mathrm{C} 4$ quiet time operations (black), and in correspondence of the injection of the highest level of acoustic noise during the test (green or light gray): (left) angular jitter $\left(\tilde{\epsilon} \equiv \tilde{\theta} / \theta_{\infty}\right)$ of the beam entering the $\mathrm{MC}$ with respect to its optical axis, and its cumulative rms (dashed line); (right) power fluctuations of MC transmitted beam $(\tilde{P})$.

fluctuations of the transmitted beam, which may contain both linear and quadratic terms. Indeed, the transmitted beam power shows a non null and different coherency pattern with both linear and quadratic beam lateral and angular fluctuations: quadratic terms dominate above $500 \mathrm{~Hz}$ and linear terms below (Figure $5 e$ and $f$ ).

\subsection{Beam jitters and power fluctuations}

In the most general case $[14,15]$ the beam enters MC cavity with angular $\left(\epsilon \equiv \theta / \theta_{\infty}\right)$ and translational jitters $\left(\beta \equiv a / w_{0}\right)$, normalized to beam divergence $\left(\theta_{\infty}\right)$ and waist $\left(w_{0}\right)$ :

$$
\epsilon=\epsilon_{s}+\epsilon(t) \quad \text { and } \quad \beta=\beta_{s}+\beta(t)
$$

where, with $\epsilon_{s}$ and $\beta_{s}$ we indicate the static (or low frequency, i. e. $<10 \mathrm{~Hz}$ ) parts, with $\epsilon(t)$ and $\beta(t)$ the high frequency ones.

In the ideal case $\epsilon(t)$ and $\beta(t)$ are filtered by the optical cavity, while $\epsilon_{s}$ and $\beta_{s}$ should be compensated by the MC alignment. Residual jitters cause a perturbation of the MC transmitted beam in amplitude and phase, as:

$$
\Psi_{\text {trans }} \approx \Psi_{00}\left(1-\frac{1}{2}\left(\epsilon^{2}+\beta^{2}\right)+2 i \epsilon \beta\right) .
$$

The first term gives rise to fluctuations of the power of the transmitted beam which contain both linear and quadratic terms, as:

$$
\tilde{P}_{\text {trans }} \approx \epsilon_{s} \tilde{\epsilon}+\beta_{s} \tilde{\beta}+\tilde{\epsilon}^{2}+\tilde{\beta}^{2} .
$$

During the acoustic noise injection we measured: $\epsilon_{s} \approx 5 \times 10^{-3}, \tilde{\epsilon} \approx 10^{-5} / \sqrt{\mathrm{Hz}}$ (Figure $4 a$ ) and similar values for $\beta_{s}$ and $\tilde{\beta}$. 

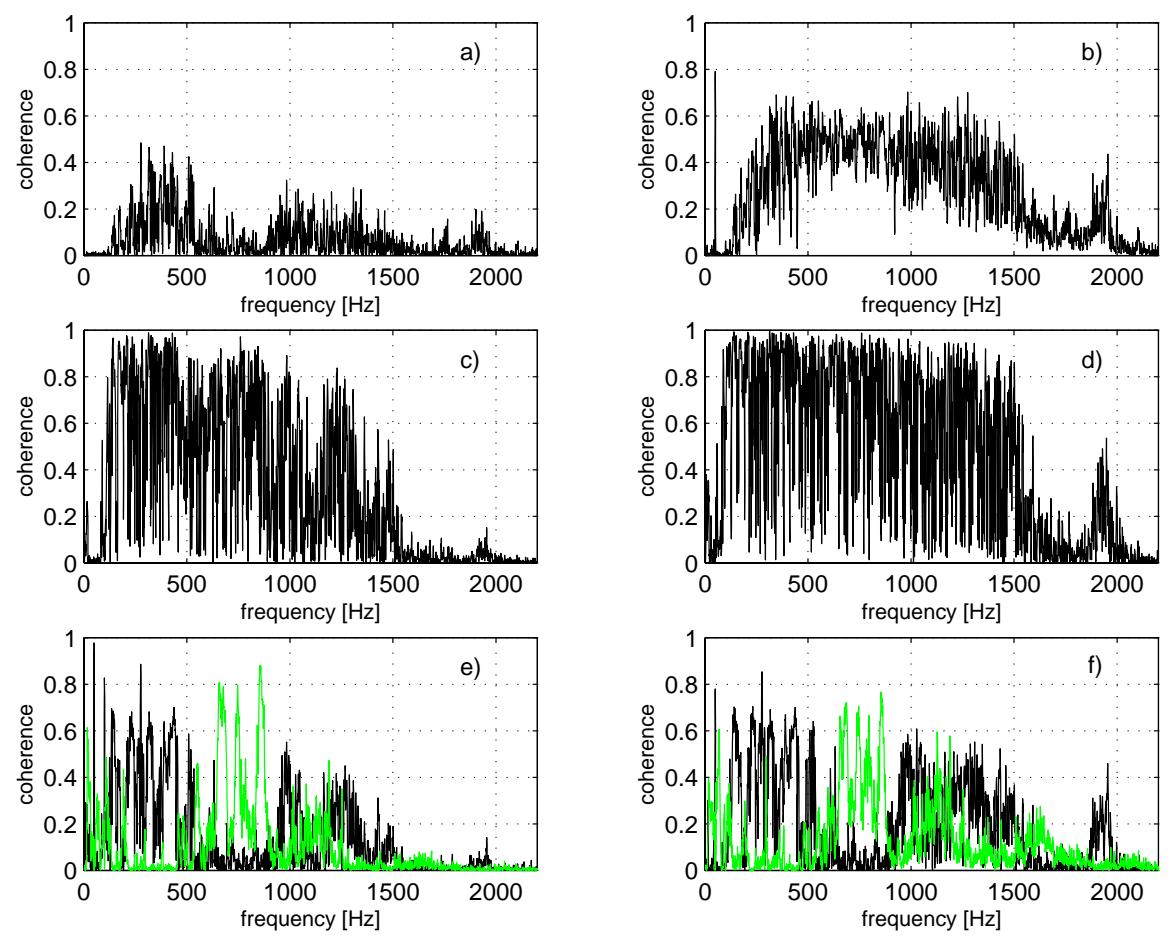

Figure 5. Coherency functions of some signals during the acoustic test: a) dark fringe and laser room microphone; b) dark fringe and power of the beam transmitted by the MC; c) laser room microphone and signal measuring the vertical misalignment of the MC optical axis $(a(t))$; d) laser room microphone and signal measuring the angular misalignment of the MC optical axis $(\theta(t))$; e) power of the beam transmitted by MC and $a(t)$ (black) and $a^{2}(t)$ (green or light gray); f) power of the beam transmitted by MC and $\theta(t)$ (black) and $\theta^{2}(t)$ (green of light gray). Each coherence plot $(0.5 \mathrm{~Hz}$ resolution) is obtained with $200 \mathrm{~s}$ of data averaging over $\Delta T=2 \mathrm{~s}$ long data chunks. A de-trend procedure is first applied to $a(t)$ and $\theta(t)$ signals, which consists in subtracting the signal's mean value from each $\Delta T$ data chunk.

\subsection{Estimate of the power noise contribution to ITF sensitivity}

Beam power fluctuations of the MC transmitted beam enter the interferometer, producing phase noise in the dark fringe signal as:

$$
\phi_{n}(t)=\Delta L \frac{\delta P(t)}{P(t)} \quad \text { or } \quad \phi_{n}(t)=L_{L F}(t) \frac{\delta P(t)}{P(t)}
$$

where, $\Delta L$ measures the rms static offset from the dark fringe condition, and $L_{L F}(t)$ is the low frequency part (i.e. $<10 \mathrm{~Hz}$ ) of the dark fringe signal [13] $\dagger$. The second model provides a more accurate evaluation which accounts for the large low frequency fluctuations of the dark fringe signal.

The contribution to the ITF displacement sensitivity is shown in Figure 6 . Beam power noise, excited by seismic/acoustic noise in the laser room, is the major

$\dagger$ In $\mathrm{C} 4$ Virgo-recombined the rms displacement from the dark fringe condition was $\Delta L \approx 3 \times 10^{-11} \mathrm{~m}$ accumulated mainly below $10 \mathrm{~Hz}$. 
contribution to the measured $\mathrm{C} 4$ Virgo-recombined dark fringe noise between $\approx 100$ $\mathrm{Hz}$ and $500 \mathrm{~Hz}$. The dark fringe noise between $\approx 500 \mathrm{~Hz}$ and $1500 \mathrm{~Hz}$, which is as well excited by the same environmental noise sources, is only partially explained by power noise. Here another kind of noise contributes, which needs further investigation.

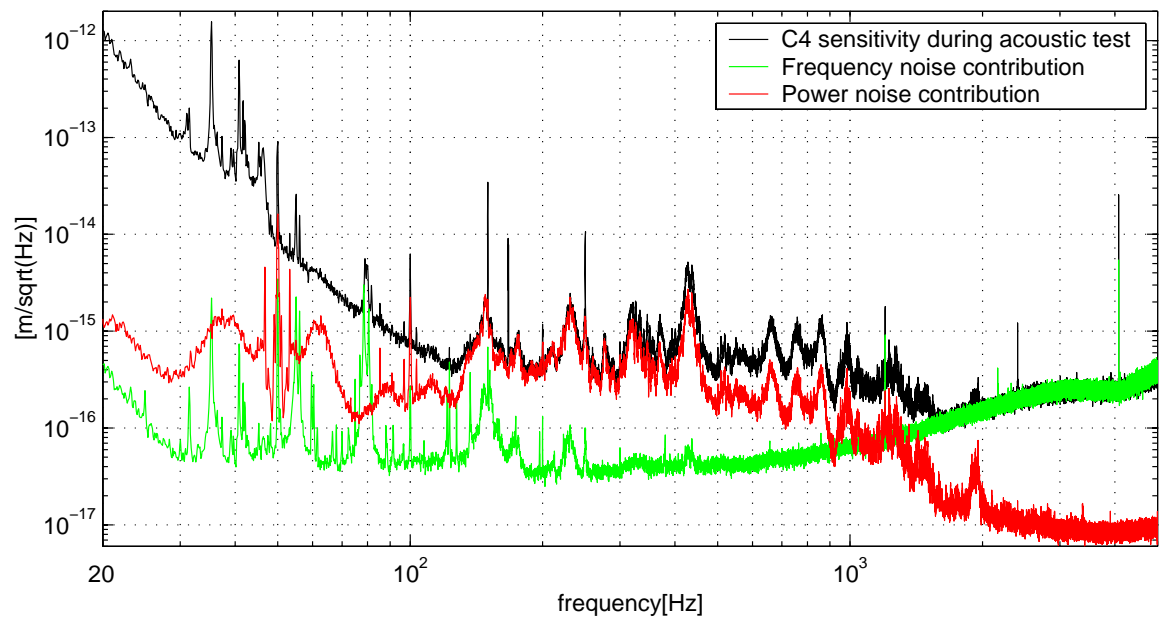

Figure 6. Virgo-recombined sensitivity during C4 acoustic test (black curve). Superimposed are the estimated frequency noise (green or light gray) and power noise (red or dark gray) contributions.

\section{Conclusions}

We have described the techniques and methods we used to identify and characterize seismic/acoustic noise sources affecting the detector sensitivity during Virgo commissioning runs $\mathrm{C} 1$ to $\mathrm{C} 4$, and finally identify a path for the noise to the dark fringe signal. Effects of these sources on Virgo dark fringe reduced as the frequency noise reduced when ITF was operated in the recombined configuration. However, measurable effects were still present in $\mathrm{C} 4$. A test of acoustic noise injection performed during $\mathrm{C} 4$ demonstrated that the noise causes an increase of the angular and lateral fluctuations of the input mode cleaner optical axis and of the power fluctuations of the transmitted beam, and finally partially contributes to the ITF sensitivity as power noise.

The acoustic noise injection test proved to be a very useful diagnostic tool. We plan to repeat it systematically to test the ITF robustness throughout the remaining Virgo commissioning steps, as well to verify the effectiveness of the foreseen improvements of the acoustic and seismic isolation of the laser room and the ongoing implementation of the laser power stabilization control.

\section{References}

[1] Acernese F et al 2004 The commissioning of the central interferometer of the Virgo gravitational wave detector, AstroPart. Phys. 21 1-22.

[2] L Barsotti et al 2004 The Commissioning of the Virgo Interferometer Arms Virgo internal doc. http : //wwwcascina.virgo.infn.it/commissioning/ARMS_Commissioning_Report_v6.doc 
[3] The Virgo collaboration, L Barsotti et al 2005 Status of Virgo, Proc. of the $9^{\text {th }}$ Gravitational Waves Data Analysis Workshop, this volume.

[4] F Barone et al 2002 IEEE Trans. Nucl. Sci. 49 405-410.

[5] The Virgo collaboration, I Fiori et al 2004 Proc. of the $5^{\text {th }}$ Edoardo Amaldi Conference on Gravitational Waves Class Quant Grav 21 5433-5440.

[6] M B Priestley 2001 Spectral Analysis and Time Series, Elsevier Academic Press.

[7] The Virgo collaboration, E Cuoco et al 2005 NAP: a tool for noise data analysis, Proc. of the $9^{\text {th }}$ Gravitational Waves Data Analysis Workshop, this volume.

[8] M Bernardini et al 1997 Vuoto vol.XXVI N.1 46-50.

[9] H Trinquet 2003 Caractérisation de la source laser du détecteur d'ondes gravitationnelles VIRGO, PhD Thesis, Université de Nice Sophia-Antipolis, France.

[10] F Bondu et al 2002 Class. Quant. Grav. 19 1421-1428.

[11] G Ballardin et al 2001 Rev. Sci. Instrum. 723643.

[12] S Braccini et al 1995 Rev. Sci. Instrum. 662672.

[13] A Giazotto 1989 Interferometric detection of gravitational waves Phys. Reports 182 No.6 365424.

[14] E Calloni et al 1997 Effects of misalignment and beam jitter in Fabry-Perot laser stabilization, Opt. Comm. 142 50-54.

[15] F Barone et al 1996 Effects of misalignment and beam jitters in interferometric gravitational wave detectors, Phys. Lett. A 217 90-96. 